\title{
Uncontroversial Default Logic
}

\author{
Paolo Liberatore ${ }^{1}$
}

\begin{abstract}
Many variants of default logics exist. Two of the main differences among them are the choice between local or global consistency and the choice of accepting maximally successful sets of defaults. Proving a result that is valid in all variants amounts to showing either a proof that holds for all semantics or a different proof for each semantics. In this paper, we characterize theories that do not depend at all on what makes the semantics different. A result that is proved on such theories holds not only for all considered semantics, but also on any other semantics that differs on the classical one because of the two choices. These theories are also of interest for practical applications of default logic, as an implemented system should be able to detect (for example, to warn the user) any theory whose semantics is debatable.
\end{abstract}

\section{INTRODUCTION}

Since the introduction of default logic [11] several variants have been introduced, for example, justified default logic [9], constrained default logic [12, 5], rational default logic [10], cumulative default logic [3], prioritized default logic [4], disjunctive default logic [6], etc. Some of these variants extend the original syntax, for example, disjunctive default logic, or require some additional data, like prioritized default logic. Nevertheless, most variants use the same syntax of the original Reiter's definition, and aim at overcoming its drawbacks.

Some semantics for default logic depend on how they answer to two questions: if the application of a default leads to inconsistency, should we insist on applying the default or not? and, should we enforce joint consistency of all justifications and consequences of the defaults? Three variants of default logic differ from the classical one because of these two choices, namely, justified default logic, constrained default logic, and rational default logic.

In this paper, we study uncontroversial default theories. Roughly speaking, a theory is uncontroversial if these two questions do not arise at all. Therefore, the above semantics agree on such theories. Uncontroversial theories are important for two reasons:

1. some kinds of results proved on them are valid regardless of the choice of the semantics; namely, this holds for results about the existence of theories with some properties, e.g., hardness results;

2. finding out that a theory is controversial allows for pointing out that its interpretation depends on the chosen semantics; e.g., an implemented system can warn the user that the evaluation of the theory is not unique.

Controversiality is not defined in terms of the four semantics cited above. A theory is not defined controversial if these four semantics

\footnotetext{
1 Dipartimento di Informatica e Sistemistica, Università di Roma "La Sapienza", via Salaria 113, 00198, Rome, Italy.
}

disagree on it. It is defined uncontroversial if it makes the two choices irrelevant. From this definition, we then prove that uncontroversial theories are actually evaluated in the same way by the classical, justified, constrained, and rational semantics. This result confirms that our formal definition of uncontroversiality formalizes what uncontroversiality intuitively is: the property of not generating disagreement. This is further confirmed by another theorem, stating that controversial theories are interpreted differently by two semantics at least.

An important consequence of this approach is that we can define the set of semantics that agree on uncontroversial theories. These semantics are independent on the choice of global/local consistency and forced application. We call these semantics consonant. If an existence (e.g., hardness) result is proved using only uncontroversial theories, it holds for any consonant semantics, and not only for the ones that have been defined so far.

Two complexity results are given in this paper: first, we prove that the complexity of model checking for all consonant semantics is $\Sigma_{2}^{p}$ hard; second, checking whether a theory is controversial is $\Sigma_{2}^{p}$ complete. The first result extends the one by Liberatore and Schaerf [8] to all consonant semantics.

The paper is organized as follows: in the next section, we summarize our notations and the definition of the semantics of default logic used in the rest of the paper; we then define uncontroversial theories; another section is devoted to consonant semantics (semantics that agree on all uncontroversial theories) and to discriminating semantics (which disagree on any controversial theory); we then analyze the relationship between controversiality and extensions. Finally, we report on the complexity analysis.

\section{PRELIMINARIES}

In this section, we define the variants of default logic we consider. A default is a structure composed of three propositional formulae, written $\frac{A: B}{C}$. The formulae $A, B$, and $C$ are called the precondition, the justification, and the consequence of the default, respectively. Technically, we assume that each default has a single justification, rather than a set of justifications. A default theory is a pair $\langle D, W\rangle$, where $D$ is a set of defaults and $W$ is a propositional formula.

Given a default $d$, its parts are denoted by $\operatorname{prec}(d), j u s t(d)$, and $\operatorname{cons}(d)$. We use the operational definition of default logics [2], which is based on sequences of defaults with no duplicates (processes). If $\Pi$ is a process, we denote by $\Pi[d]$ the sequence of defaults preceeding $d$ in $\Pi$, and by $\Pi \cdot[d]$ the sequence obtained by adding $d$ at the end of $\Pi$. We extend the notation from defaults to sequences, so that $\operatorname{cons}(\Pi)$ is the conjunction of all consequences of the defaults in the sequence $\Pi$ plus the background theory $W, \operatorname{prec}(\Pi)$ is the conjunction of all preconditions, and $j u s t(\Pi)$ is the conjunction of all justifications.

Implication is denoted by $\models, \top$ indicates (combined) consistency, 
and $\perp$ indicates inconsistency. For example, $A \top B$ means that $A \wedge B$ is consistent, while $A \perp B$ means that $A \wedge B$ is inconsistent.

Default logic can be defined in terms of the selected processes, that are the sequences of defaults that are considered applicable by the semantics [1]. A default $d$ is locally applicable in a sequence $\Pi$ if $\operatorname{cons}(\Pi) \models \operatorname{prec}(d)$ and $\operatorname{cons}(\Pi) \top j u s t(d)$. Global applicability also requires cons $(\Pi \cdot[d]) \top j u s t(\Pi \cdot[d])$. Each semantics defines the sequences of defaults that are applied in a particular theory. Formally, the definitions are as follows:

Reiter: a selected process is a sequence of defaults $\Pi$ such that $\operatorname{cons}(\Pi[d]) \models \operatorname{prec}(d)$ for each $d \in \Pi$, that $\operatorname{cons}(\Pi) \top j u s t(d)$ for each $d \in \Pi$ (success) and no other default $d^{\prime} \notin \Pi$ is locally applicable in $\Pi$ (closure);

Justified: a selected process is a maximal sequence of defaults $\Pi$ such that $\operatorname{cons}(\Pi[d]) \mid=\operatorname{prec}(d)$ and cons $(\Pi) \top j u s t(d)$ for each $d \in \Pi$;

Constrained: a selected process is a maximal sequence of defaults $\Pi$ such that $\operatorname{cons}(\Pi[d]) \models \operatorname{prec}(d)$ for each $d \in \Pi$ and $\operatorname{cons}(\Pi) \top j u s t(\Pi)$;

Rational: a selected process is a sequence of defaults $\Pi$ such that $\operatorname{cons}(\Pi[d]) \models \operatorname{prec}(d)$ for each $d \in \Pi$, cons $(\Pi) \top j u s t(\Pi)$, and for no $d \notin \Pi$ both cons $(\Pi) \models \operatorname{prec}(d)$ and cons $(\Pi) \top j u s t(\Pi$. [d]) hold.

When more than one default is applicable, we have to select which one to apply, leading to different paths of applications of defaults. Application depends on the semantics: namely, we can decide to apply a default if it is locally consistent, or only if it is globally consistent. Moreover, when all applicable defaults lead to a sequence of defaults we do not accept (because it is not locally or globally consistent), we have the choice of selecting the process as is, or to rejecting it. These are, formally, the two choices of global/local consistency, and the acceptance of maximally successful processes.

\section{UNCONTROVERSIAL DEFAULT THEORIES}

The idea is to characterize default theories whose interpretation is not only independent on some specific semantics, but is not affected at all by the question of local/global consistency and by the question of accepting maximal processes. To this aim, we consider two definitions of process, one allowing as many sequences of defaults to be regarded as processes, the other one being so restrictive that only sequences for which no doubt exists are regarded as processes. The rationale is that, if all process of the first kind are also of the second kind, then no debate about the semantics of the theory is possible.

Definition 1 A general process is a sequence of defaults $\Pi$ such that, for each default $d$ of $\Pi$, it holds:

1. $\operatorname{cons}(\Pi[d]) \models \operatorname{prec}(d)$; and

2. $\operatorname{cons}(\Pi) \top j u s t(d)$.

The aim of this definition is to capture any sequence of defaults that is even remotely considered acceptable. As a result, we make only a local consistency checking, that is, we check only whether $\operatorname{cons}(\Pi) \top j u s t(d)$ and not whether cons $(\Pi) \top j u s t(\Pi)$, as the latter restricts the number of possible processes. Moreover, we do not force processes to be closed, so a default may be applicable in a general process without being part of it. We will however enforce maximality of such processes to avoid "harmless" applicable defaults to be excluded from processes. It is easy to see that maximal general processes are the ones selected by justified default logic.
This definition is as general as possible to capture the processes that are selected by any semantics. More precisely, any selected process should be a maximal general process. The following definition captures the processes that are selected regardless of whether we require local or joint consistency, or whether we accept maximally successful processes.

Definition 2 An uncontroversial process is a general process $\Pi$ with two additional properties:

1. $\operatorname{cons}(\Pi) \top j u s t(\Pi)$; and

2. if $d \notin \Pi$ then either cons $(\Pi) \not \models \operatorname{prec}(d)$ or cons $(\Pi) \perp j u s t(d)$.

The first rule enforces joint consistency of all justifications with all consequences; the second rule forbids any other default to be applicable (using local applicability).

This definition aims at capturing as few processes as possible. Namely, since we want to be sure that the choice of local and global consistency has no effect, we only select processes that are jointly consistent. Since we do not want the choice of maximally successful processes to have any effect either, we only consider processes that are maximal because no other default is applicable, and not because all applicable defaults lead to failure.

Summarizing, we have defined general processes to be sequences of defaults that a very liberal semantics selects; we have then defined uncontroversial processes to be processes that are selected regardless of the choice of local/global consistency and maximally successful processes. General processes are not necessarily closed nor maximal. However, it is easy to prove the following theorem.

Theorem 1 Uncontroversial processes are maximal general processes.

We now define the theories that are not subject to debate.

Definition 3 An uncontroversial theory is a default theory whose general processes are initial segments of uncontroversial processes.

By Theorem 1, this definition can be rephrased as: all maximal general processes of an uncontroversial theory are uncontroversial processes. Since a semantics selects processes among the maximal general ones, if all of them are uncontroversial, then we can conclude that the whole theory is uncontroversial as well: this is the rationale behind this definition.

Example 1 The following example formalizes an excerpt of the call for paper of this conference: "at least one author of each accepted paper is required to attend the conference to present the paper." From this sentence, one can infer that, by default, a paper will be presented. This is a default rule, as it only holds if we can consistently assume that at least one of the authors will attend the conference. Let us therefore consider a paper that has two authors $A$ and $B$. We can then encode this example as the default theory $\langle D, W\rangle$, where $W=\{$ accepted $\}$, and $D=\left\{d_{1}, d_{2}\right\}$, where $d_{1}$ and $d_{2}$ are defined as follows.

$$
\begin{aligned}
d_{1} & =\frac{\text { accepted }: \text { A_attends }}{\text { presentation }} \\
d_{2}= & \frac{\text { accepted }: \text { B_attends }}{\text { presentation }}
\end{aligned}
$$

The maximal general processes are $\left(d_{1}, d_{2}\right)$ and $\left(d_{2}, d_{1}\right)$ : indeed, the preconditions of both $d_{1}$ and $d_{2}$ are already implied by $W$, and 
their justifications cannot be contradicted. Both processes are uncontroversial. Indeed, the conjuction of the justifications of $d_{1}$ and $d_{2}$ are consistent with their consequences and $W$; therefore, the first condition of uncontroversiality is satisfied. The second condition is ensured by the fact that these processes contain all defaults.

We also define the opposite concept of controversiality: a controversial process is a process that is not uncontroversial, and a controversial theory is a theory that is not uncontroversial. Note that a theory is controversial if and only if it contains a controversial process, but can nonetheless contain uncontroversial processes as well.

Example 2 The default theory of the above example can be made controversial by simply modifying the theory $W$. Let us assume that the authors of the paper cannot both attend the conference to present the paper due to another duty they have. This fact can be formalized by modifying $W$ as follows:

$$
W=\left\{\text { accepted, } \neg \text { A_attends } \vee \neg B \_ \text {attends }\right\}
$$

The resulting default theory $\langle D, W\rangle$ is controversial. This can be proved by showing a maximal general process that is not uncontroversial. Consider the process $\left(d_{1}, d_{2}\right)$. This is a general process, as the consequences of its defaults are consistent with $W$ and with each justification. On the other hand, they are not consistent with the union of all justifications, and is therefore not uncontroversial.

While the theory of this second example is controversial, the same domain can be formalized by the uncontroversial theory that explicitely contains the fact that the presence of and author is what makes the presentation possible, that is,

$$
\begin{aligned}
D=\left\{\begin{array}{l}
\text { accepted }: \text { A_attends } \\
\text { A_attends }
\end{array}\right. & \frac{\text { accepted }: \text { B_attends }}{\text { B_attends }}, \\
& \frac{\text { accepted } \wedge \text { A_attends }: \text { presentation }}{\text { presentation }}, \\
& \left.\frac{\text { accepted } \wedge \text { B_attends }: \text { presentation }}{\text { presentation }}\right\}
\end{aligned}
$$

The conclusions we can draw from the information of this domain are quite clear: we know that exactly one author will attend and present the paper. Nevertheless, the previous formalization is interpreted differently by some semantics. In this case, controversiality is caused by the encoding of the domain, and not by the domain itself. Checking controversiality can be therefore useful for verifying whether a particular encoding may cause such problems.

\section{CONSONANT SEMANTICS}

Consonant semantics are those differing from the original one only for the two choices of global/joint consistency and acceptance of maximal processes. The formal definition is based on the general and uncontroversial processes.

Definition 4 A semantics of default logic is called consonant if and only if the selected processes of an arbitrary theory form a subset of its general processes and a superset of its uncontroversial processes.

This definition captures the semantics of default logic that agree on uncontroversial theories. We do not insist on the converse: two semantics may agree on a theory containing a controversial process.
Theorem 2 The processes and the extensions of an uncontroversial theory are the same for all consonant semantics.

Classical, justified, constrained, and rational default logic are actually consonant, and therefore agree an all uncontroversial processes. We can indeed prove that uncontroversial processes are selected by all semantics, and that a process for an arbitrary semantics is a general process. We give the proof for classical default logic as an example.

Lemma 1 An uncontroversial process is a selected (i.e., closed and successful) process for classical default logic.

Proof. Let $\Pi$ be an uncontroversial process. We prove that it is a closed and successful process under the classical semantics.

1. The first condition is that any default $d$ must be applicable in $\Pi[d]$. This amounts to proving that its precondition is implied by cons $(\Pi[d])$, and that cons $(\Pi[d])$ is consistent with the justification of $d$. The first point of the definition of (general) process is indeed that $\operatorname{cons}(\Pi[d]) \models \operatorname{prec}(d)$. The second condition cons $(\Pi[d]) \top j u s t(d)$ is a consequence of the second point of the definition of process, that is, cons $(\Pi) \top j u s t(d)$.

2. The second condition is that the sequence of defaults must be successful, that is, cons $(\Pi) \top j u s t(d)$ for any default $d \in \Pi$. This is not implied by the previous condition, as applying a new default can contradict the justification of a previous one. However, the second point of the definition of general process is that $\operatorname{cons}(\Pi) \top j u s t(d)$ for any $d \in \Pi$.

3. The third condition is the closure: no default $d \notin \Pi$ is applicable in $\Pi$. For this condition, we have to use the assumption of uncontroversiality. Its second point is that, for any $d \notin \Pi$, either $\operatorname{cons}(\Pi) \not \neq \operatorname{prec}(d)$ or cons $(\Pi) \perp j u s t(d)$. But the default $d$ is applicable in $\Pi$ if and only if both conditions are true.

Lemma 2 Any selected (i.e., closed and successful) process under the classical semantics is a general process.

Proof. Let $\Pi$ be a closed and successful process. We prove it is a general process. This amounts to showing that cons $(\Pi[d]) \models \operatorname{prec}(d)$, and that cons $(\Pi) \top j u s t(d)$ for any $d \in \Pi$.

1. The first condition is implied by the first point of the definition of classical default logic, which is: $d$ is applicable in $\Pi[d]$. Indeed, this includes the condition cons $(\Pi[d]) \models \operatorname{prec}(d)$.

2. The second condition is that cons $(\Pi) \top j u s t(d)$. This is exactly the successfulness of $\Pi$.

This proves that any process selected by the classical semantics is a general process.

Together, these two lemmas imply that the extensions of an uncontroversial theory are exactly the consequences of its uncontroversial processes. A similar statement can be proved for the other considered semantics (the proof is omitted due to the lack of space.)

Theorem 3 Classical, constrained, justified, and rational default logics are consonant.

The fact that classical, constrained, justified, and rational default logics are consonant proves that the definition of uncontroversiality reflects its intuitive meaning: uncontroversial default theories are 
given the same interpretation (i.e., they have the same extensions) by these different logics.

What makes uncontroversiality interesting is that we started with a definition that characterizes the theories that are not affected by the choices of local/global consistency and maximality, and proved that this actually implies the agreement among the four considered semantics. While this result confirms our intuition, it cannot be used as a definition of uncontroversiality. Indeed, if we decide that any definition that satisfies this property is a reasonable definition of uncontroversiality, then even weird definitions may work. As an example, we could then define uncontroversial a theory $\langle D, W\rangle$ if and only if $W$ is inconsistent. Such a definition work, but does not fully capture all theories that we want to regard as uncontroversial.

\section{DISCRIMINATING SEMANTICS}

Discriminating semantics allows for telling whether a theory is controversial by behaving differently on it.

Definition 5 A set of semantics is called discriminating if any controversial theory is assigned a different set of selected processes by at least two semantics in the set.

If we can prove that a set of reasonable semantics is a discriminating set, we can conclude that our definition of controversiality is founded, in the sense that we are not calling controversial a theory whose meaning is not actually controversial. This is what we are actually going to show: a controversial theory is interpreted differently by at least two semantics among classical, justified, and constrained. Rational default logic is not needed to form a discriminating set.

Lemma 3 If $\Pi$ is a maximal general process that is controversial, then it is selected by one semantics among classical, justified, and constrained, but not by another one.

Proof. Let $\Pi$ be a maximal general process that is not uncontroversial. We have already observed that maximal general processes are exactly those selected by justified default logic. We prove that $\Pi$ is not selected by at least one other semantics.

By definition, a process is controversial if and only if either global consistency does not hold, or some other default is (locally) applicable. In the first case, cons $(\Pi) \perp j u s t(\Pi)$, which implies that $\Pi$ is not selected by constrained default logic. In the second case, there exists a default $d \notin \Pi$ that is locally applicable in $\Pi$, which implies that $\Pi$ is not a closed process according to the classical semantics, and is therefore not selected by it.

Since uncontroversial processes are maximal general processes, if a theory has a general process that is not uncontroversial, it has at least a maximal general process that is controversial, and vice versa. The last lemma therefore applies to all controversial theories. We can conclude that the interpretation of a controversial theory depends on the semantics.

Theorem 4 Classical, justified, and constrained default logic form a set of discriminating semantics.

\section{PROCESSES AND EXTENSIONS}

The definition of uncontroversial theories is based on processes, rather than extensions. From this definition we proved that, if a theory is controversial, then two semantics selects different processes.
This result, however, does not forbid the overall evaluation of the theory to be the same, as two different processes may lead to the same extension. This is actually the case for some controversial default theories. Let $\langle D, W\rangle$ be the default theory defined by $W=\emptyset$ and $D$ is $D=\{: x / y, y: \neg x /$ true $\}$. This theory has exactly one maximal general process: the sequence of both defaults. However, this process is not uncontroversial, as the set of all justifications is $\{x, \neg x\}$, which is not consistent.

The selected processes of this default theory depend on the chosen semantics. Indeed, both classical and justified default logic take $D$ to be a process, while constrained default logic selects the first default only, as the second one lead to global inconsistency. Nevertheless, this difference in the selected processes does not lead to different sets of extensions. Indeed, the second default has no consequence; thus, its presence in the process is irrelevant to the set of extensions.

Although this theory has different processes depending on the semantics, its sets of extensions are the same. In general, this may happen because the same extensions can be produced by different processes. Therefore, it may be that a semantics selects the processes $\Pi_{1}$ and $\Pi_{2}$, while another one selects $\Pi_{3}, \Pi_{4}$, and $\Pi_{5}$, but the set of extensions is the same because the consequences of $\Pi_{1}$ are equivalent to those of $\Pi_{3}$ and $\Pi_{4}$, and the consequences of $\Pi_{2}$ and $\Pi_{5}$ are equivalent.

The question is: can we still call controversial a theory whose evaluation is the same in all semantics? After all, the three considered semantics agree on $\langle D, W\rangle$. We remark that the concept of controversiality is not derived from that of "having different extensions". Rather, its definition is based on the concept of "being evaluated differently", and having different processes means that the semantics behave differently, even if the final result is the same. Some definitions of extensions (namely, constrained extensions) also include the set of justifications used for deriving the set of consequences. If this is the case, processes reflect the meaning of theories better than extensions (intended as sets of consequences) do.

There are other reasons for choosing the set of processes to represent the semantics of theories, rather than using the set of extensions. Let us assume, for example, that we regard $\langle D, W\rangle$ as uncontroversial only because it has the same set of extensions. Then, adding $z$ to the consequences of the second default, the theory is made controversial. This is quite unintuitive: $z$ is not even mentioned in the original theory $\langle D, W\rangle$, so it should not conflicts with any previous default; thus, it should not have any consequence on the theory being controversial or not. Intuitively, the controversiality status of a default theory should not change when something irrelevant (i.e., not even mentioned in some other place) is added to either the justification or the consequences of a single default, which instead happens when controversiality is defined in terms of extensions.

Another good reason for defining controversiality in terms of processes rather than extensions is that the justifications are, in some settings, used. For example, lemma defaults [12] include the justifications used in the process of derivation. Defining controversiality in terms of extensions leads to the unintuitive result that the lemmas of an uncontroversial theory may depend on the semantics.

\section{COMPLEXITY}

In this section, we report on the complexity analysis of controversiality and consonant semantics. Namely, we show an hardness proof involving only uncontroversial theories: this result holds for any consonant semantics. We also study the complexity of deciding whether a theory is controversial, as the possible disagreement of the seman- 
tics is an important point to emphasize when evaluating a default theory.

$\Sigma_{2}^{p}$-hardness can be proved for the complexity of model checking, for all consonant semantics. This means that the problem is $\Sigma_{2}^{p}$-hard for any semantics for default logic that can be proved to be consonant. This extends a result by Liberatore and Schaerf [8].

Theorem 5 If a semantics is consonant, its complexity of model checking is $\Sigma_{2}^{p}$-hard. This holds even if the theory is seminormal and precondition-free.

Having proved a lower bound for model checking for any consonant default semantics, the next natural question is whether an upper bound can be given. This is however impossible: the definition of consonant semantics does not constrain them enough to disallow encoding arbitrarily hard problems, such as the halting problem. This can be done even for very simple theories: for example, there are consonant semantics that selects processes for $\left\langle\left\{\frac{b}{c}\right\}, b \wedge \neg c \wedge W^{\prime}\right\rangle$ according to whether formula $W^{\prime}$ (which contains neither $b$ nor $c$ ) represents an always-terminating Turing machine, according to some encoding. Model checking becomes as hard as the halting problem for such semantics. This semantics is clearly artificial, but it nevertheless shows that no upper bound can be given for all consonant semantics.

The analysis of query answering is much simpler. Since query answering is $\Pi_{2}^{p}$-hard for classical default logic even for normal theories [7], and these theories are uncontroversial, we can conclude that the problem is $\Pi_{2}^{p}$-hard for all consonant semantics.

Let us now turn to complexity of checking whether a theory is controversial. As already remarked in the Introduction, any implementation of default logic should detect controversiality of theories, as such theories do not have an unique interpretation.

Theorem 6 Checking controversiality of a theory is $\Sigma_{2}^{p}$-complete.

\section{CONCLUSIONS}

We have given a characterization of default theories whose evaluation is not subject to how the semantics choose between local or joint consistency, or whether it accepts maximally successful sets of defaults. The definition of uncontroversial processes is surprisingly simple, considering that it captures exactly the theories that have the same evaluation under classical, justified, constrained, and rational semantics, and any controversial theory is instead evaluated differently by two semantics in this set, at least.

The concept of uncontroversial theories induces some related concepts. Consonant semantics generalize the ones cited above: they are the semantics that agree on uncontroversial theories. Another extension is that of discriminating sets, which are composed of semantics that evaluate differently all controversial theories.

The last section of the paper is the complexity analysis. First, we have given a complexity result for all consonant semantics; second, we have studied the complexity of checking whether a default theory is controversial. The first result is interesting, as it shows an application of controversiality: a proof of hardness involving only uncontroversial theories holds for any consonant semantics. This way, it is possible to give a single simple proof for all consonant semantics. The second result is of interest, as a system that implements default reasoning should detect controversiality as a sign of possible mistakes, since the interpretation of controversial theories is not unique.

Some problems are left open. In particular, there are semantics for default logic that have not been analyzed here, for example, cumulative default logic, prioritized default logic, disjunctive default logic, and weak extensions. Some of them require an extended syntax, but a concept of controversiality may be defined anyway.

A similar open question regards other forms of nonmonotonic reasoning for which different semantics have been given, like the closed world assumption: Is there any meaningful definition of theories that are uncontroversial to evaluate under the closed world assumption? Does it provide a sufficiently large set of theories, or only trivial ones are evaluated in the same way by all semantics? Is there any nontrivial complexity characterization for all of them? These are all open questions.

Other, more theoretical, questions are left open about consonant semantics. Namely, is there any characterization of all consonant semantics, e.g., a set of postulates that capture exactly all of them? A preliminary investigation suggests that controversiality is too weak, and other properties are required to obtain a set of meaningful postulates (for example, controversiality does not impose variable name changes to have no effect on the set of selected processes.) A similar analysis is possible for the sets of discriminating semantics: all is known is a single such set, composed of the classical, justified, and constrained semantics. We do not known whether rational semantics can replace one of them, or whether there exists a discriminating set composed of two meaningful semantics only.

\section{REFERENCES}

[1] G. Antoniou, 'A tutorial on default logics', ACM Computing Surveys, 31(3), 337-359, (1999).

[2] G. Antoniou and V. Sperschneider, 'Operational concepts of nonmonotonic logics, part 1: Default logic.', AI Review, 8(1), 3-16, (1994).

[3] G. Brewka, Nonmonotonic Reasoning: Logical Foundations of Commonsense, Cambridge University Press, Cambridge, 1991.

[4] G. Brewka, 'Reasoning about priorities in default logic', in Proc. of AAAI'94, pp. 940-945, (1994).

[5] J. P. Delgrande, T. Schaub, and W. K. Jackson, 'Alternative approaches to default logic', Artificial Intelligence, 70, 167-237, (1994).

[6] M. Gelfond, V. Lifschitz, H.Przymusinska, and M. Truszczynski, 'Disjunctive defaults', in Proc. of KR'91, pp. 230-237. Morgan Kaufmann, Los Altos, (1991)

[7] G. Gottlob, 'Complexity results for nonmonotonic logics', J. of Log. and Comp., 2, 397-425, (1992).

[8] P. Liberatore and M. Schaerf, 'The complexity of model checking for propositional default logics', in Proc. of ECAI'98, pp. 18-22, (1998).

[9] W. Lukaszewicz, 'Considerations on default logic: an alternative approach', Computational Intelligence, 4(1), 1-16, (1988).

[10] A. Mikitiuk and M. Truszczynski, 'Constrained and rational default logics', in Proc. of IJCAI'95, pp. 1509-1517, (1995).

[11] R. Reiter, 'A logic for default reasoning', Artificial Intelligence, 13, $81-$ 132, (1980)

[12] T. Schaub, 'On constrained default theories', in Proc. of ECAI'92, pp. 304-308, (1992). 